\title{
What May Be Learned about Crime in Europe (and Beyond) from International Surveys of Youth: Results from the International Self-Report Delinquency Study (ISRD3)
}

\author{
Ineke Haen Marshall ${ }^{1} \cdot$ Majone Steketee $^{2,3}$
}

Published online: 27 August 2019

(C) Springer Nature B.V. 2019

\section{Introduction}

Introducing this special issue on the ISRD requires a brief detour to the recent past (June 2013), when this journal published a special issue, Compassionate Criminology: The Legacy of Josine Junger-Tas. In that 2013 thematic issue, we celebrated the important contributions of one of the grand dames of criminology, Josine Junger-Tas (1929-2011). Josine Junger-Tas contributed in many ways to the field: she was the first editor of the European Journal on Criminal Policy and Research, she was a co-founder of the European Society of Criminology, she combined a strong commitment to policy-relevant research with an explicit and strong grounding in a moral belief that it is one's duty to do what is right- hence the conclusion that her main heritage lies in "drawing the contours of what may be called evidence-based compassionate criminology" (Marshall \& Boutellier 2013a, 2013b, 71). Josine Junger-Tas strongly believed in the proper use of method and theory, that research should be evidencebased and applied, and that one needs to have civic engagement. However, and most importantly for the present special issue, Josine also was one of the early believers in comparative work. Without her commitment to comparative criminology, the articles presented in the current special issue-drawing from a rich trove of original international data on juvenile offending and victimization - could not have been written.

Indeed, perhaps one of Josine Junger-Tas' proudest achievements was her role in launching the International Self-Report Delinquency Study (ISRD). The ISRD was created in 1990 "when a number of researchers, representing 13-mainly European - countries, came together to decide on conducting a study and to develop a common methodology" (Junger-Tas et al.

Ineke Haen Marshall

i.marshall@northeastern.edu

1 School of Criminology and Criminal Justice and Department of Sociology \& Anthropology,

Northeastern University, Boston, MA, USA

2 Verwey-Jonker Institute, Utrecht, the Netherlands

3 Erasmus University, Rotterdam, the Netherlands 
2003 , 4.) ISRD1 was reimagined later as the pilot for the second study (ISRD2), a much larger project in which more than 30 countries collaborated. Data for ISRD2 were collected between 2005 and 2007. The ISRD2 data are now archived and available for public use (Enzmann et al. 2015), and a number of books and articles have been published (see Enzmann et al. 2018, 3-4 for a selected listing). The Many Faces of Youth Crime: Comparing and Contrasting Theoretical Perspectives on Youth Crime (Junger-Tas et al. 2012) reported the main ISRD2 results. Unfortunately, Josine was unable to witness the beginning of the fieldwork for ISRD3 (December 2012), which continued to build on the foundations of ISRD1 and ISRD2, using a partly modified questionnaire allowing the testing of theories such as institutional anomie theory, procedural justice theory, and situational action theory. At the time of this writing, 32 countries have completed data collection for ISRD3. ${ }^{1}$ Preparations for ISRD4 are currently under way, with fieldwork planned to take place between 2020 and 2022.

From its beginnings, the ambition of the ISRD project has been to measure the comparative prevalence and incidence of offending and victimization, to test theories about correlates of offending and victimization in different national contexts, and to develop policy-relevant recommendations (Enzmann et al. 2018, 5). The project relies on a common research protocol, aiming to standardize questionnaire content and administration, and comparable sampling procedures in participating countries. Over the three data collection waves (ISRD1 1990-1992; ISRD2 2006-2008, ISRD3 2012-2019), the project has certainly made advances in its attempt to maximize international standardization, but there remain important questions regarding the degree to which the data collected are truly internationally comparable (Marshall 2010, Marshall \& Enzmann 2012, Enzmann et al. 2018). We share these concerns, and we are excited about ongoing attempts to critically assess the limitations of a standardized crossnational survey such as the ISRD (e.g. Rodríguez et al. 2015). We hope that the manuscripts in this special issue will illustrate that the ISRD project has come a long way since its early beginnings in the 1990s; it now provides a valuable original database which may be explored either as a merged data set (see the papers by Manzoni \& Schwarzenegger; Binik et al.; Rocca et al.; and Kammigan et al. in this issue), or to present results based on in-depth analysis of one country (De Buck \& Pauwels; Martins et al. in this issue).

\section{This Special Issue}

The first paper, by Manzoni and Schwarzenegger (2018), provides an excellent example of the kinds of insights that may be gained from careful analysis of a large international data set. Their paper explores the mechanisms involved in the often-found link between physical maltreatment and violent offending. They can do so because the ISRD3 questionnaire includes questions on physical maltreatment of children by parents, a topic of obvious practical and theoretical importance. They try to gain more insight into the cycle of violence by analyzing data from 26 countries $(n=61,040)$ to test theoretical propositions derived from three major theories (social learning, self-control, and social control/bonding theory). They first examine the mediating effect of variables derived from the three theories using the total sample, and they find a significant direct effect of maltreatment on the use of violence and indirect (mediating) effects via each of the proposed mediators; however, the mediating effect of

\footnotetext{
${ }^{1}$ See A Global Perspective on Young People as Offenders and Victims: First Results from the ISRD3 Study (Enzmann et al. 2018) for more details.
} 
self-control and deviant peers is stronger than that of bonding and moral values. They then test whether the same mediating effects found in analysis of the large data set also apply to each of the 26 individual countries, and here they find that there is great variability of direct, indirect, and total effect of maltreatment on violence across countries and within each mediator. In a sense, then, this paper illustrates how important it is to recognize and explore cross-national variability, even when there is clear empirical evidence of larger patterns of shared variance and generalizability. They conclude their paper with the tentative but intriguing observation that the negative impact of parental maltreatment may be greater in places where such parental behavior is less common.

The second paper, by Oriana Binik et al. (2019), provides an interesting analysis of one of the major sociological concepts in contemporary criminology, that of "social capital", and its role in both offending and victimization among young people. Both ISRD2 and ISRD3 include measures of this important concept, and this paper includes a comparison of the social capital scale (for 15 countries; ISRD2 $n=38,178$; ISRD3 $n=42,801$ ), showing relatively little change between the two data collection waves. Most work on social capital and collective efficacy has been done in North America, and as the authors point out, an important contribution of their work is that they expand the test of collective efficacy and social capital to a data set that includes a large variety of countries, including non-English-speaking countries $(n=55,201 ; 23$ countries). Their analysis does support the proposition that living in a neighborhood with higher levels of social capital is a protective factor - not only for delinquent involvement but also for reducing the likelihood of victimization. Consistent with the ambition of the ISRD project, the authors speculate about the policy implications of their findings. They make a strong argument for the importance of community involvement, rather than repressive measures such as zero-tolerance policies.

Concern with policy is also evident in the paper by Rocca et al. (2019). Analyzing the same sample (23 countries, $n=55,201$ ) as Binik and colleagues, they show how creative use of follow-up questions (a new development introduced in ISRD3) can provide new insights into a much-studied topic (alcohol and cannabis use among young people). Not surprisingly, they find that both alcohol and cannabis use is not uncommon among the youth sample but that it is more prevalent among youth who commit offenses. In their paper, they attempt to disentangle the criminogenic effect of alcohol consumption and cannabis use, respectively. They analyze a subsample of youth who admitted to vandalism, carrying a weapon, assault, and group fighting, and who answered the online follow-up question: "Were you under the influence of alcohol or drugs when you did this?" Finding that delinquency under the influence of substances such as alcohol or cannabis is not very high, they conclude that delinquency should not be understood as a direct physio-pharmaceutical effect of substance use, but rather as rooted in "a generically deviant behavioral dimension, in which the various external and internal factors concur to trigger both behaviors, which, in turn, feed each other reciprocally" (page xx). Prevention polices should, therefore, focus on social context, family environment, and personal characteristics, rather than on substance use.

The paper by Kammigan et al. (2018) is only tangentially concerned with the substantive issues of crime and victimization. Instead, this paper is an ambitious attempt to deal with an important methodological problem in self-report surveys (i.e. social desirability bias), which is likely amplified in a cross-national study such as the ISRD. Within this context, we would like to note that ISRD3 has included several measures to try to deal with potential validity and reliability threats, such as including a response integrity question based on a variant of the cross-wise model (Yu et al. 2008) as a means of statistically correcting for systematic bias 
(Enzmann et al. 2018). The present paper uses questions asking about willingness to underreport drug use ("Imagine you had used cannabis - do you think you would have said so in the questionnaire?") and overreporting of drug use (measured by asking students whether they had ever used the nonexistent drug relevin). Recognizing that individuals' responses to questionnaires should be viewed as social behavior, Kammigan and colleagues conceptualize under- or overreporting of drug use as instances of general moral rule-breaking behavior which may be analyzed through the framework of situational action theory (SAT). The goal of their study is to explain individual- and country-level differences in socially desirable responding by the key constructs of SAT, and they accomplish this by analysis of 67,475 students in grades 7 through 9 from 29 countries. Although the researchers conclude that SAT appears to be a suitable candidate for explaining socially desirable responding, they caution one to be aware of the complexity of trying to test SAT (e.g. measurement issues related to morality). The paper speculates about the consequences of their findings for the use of self-report methodology, recognizing that some factors that appear to influence under- or overreporting are simply beyond the control of the researchers.

As the preceding paragraphs show, a large international data set can provide compelling methodological and theoretical insights based on analysis of several combined samples drawn in different national contexts. On the other hand, researchers may also learn a great deal by indepth analysis of the responses of their own country-level sample. The last two papers in this special issue analyze such country-based samples, respectively, from Belgium (De Buck \& Pauwels 2018) and Portugal (Martins et al. 2018). De Buck and Pauwels base their analysis on the Belgian ISRD3 survey, a paper-and-pencil survey among 4745 secondary school pupils in four cities. They test the routine activities theory (RAT), with youth's intention to shoplift as dependent variable. This variable is not part of the larger ISRD survey, but it illustrates how the ISRD3 project allows national participants to add a national module, adding questions that are not part of the standardized core questionnaire. Because the Belgian ISRD research team was particularly interested in studying criminal intent, they adopted questions that allowed the use of the scenario method to investigate underlying motivations governing human evaluations, judgments, and decision making. They find that the empirical results are very much in line with their conceptual model, and they present implications for crime prevention and policy.

The last paper, by Martins and colleagues (Martins et al. 2018), presents the results of the ISRD3 project in Portugal. As the authors write, this research "is a contribution to the epidemiology of youth victimization in Portugal, as the first large study of this subject in this country" (Martins et al., p? in this issue). The Portuguese sample has a wider age range (12-21 years) than the other ISRD3 samples (12-16 years); the focus of this paper is victimization (rather than offending) and its demographic and socioeconomic correlates among Portuguese youth $(n=4048)$. Noteworthy findings are the relatively low level of victimization reported, and the high number of young people who were victims of parental violence. Because of the relatively low importance of sociodemographic variables as predictors of victimization, the authors argue for further exploring stronger theoretical models.

Josine Junger-Tas, the pioneer of the ISRD project, envisioned the International SelfReport Delinquency (ISRD) project as an ever-evolving internationally collaborative endeavor producing robust self-report data on offending and victimization, with a strong methodological and theoretical foundation, building knowledge for policy and prevention. These six papers represent but a sample of analyses that may be performed with the ISRD3 data. "But already the evidence is in: the design and execution of this major research project provides information that will enrich criminology for years to come" 
(Gottfredson 2018, viii). This resounding endorsement of the ISRD project makes us proud to be continuing the groundbreaking work started by Josine Junger-Tas some 30 years ago. Preparations for ISRD4 are currently under way!

\section{References}

Binik, O., Ceretti, A., Cornelli, R., Schadee, H., Verde, A., \& Gatti, U. (2019). Neighborhood Social Capital, Juvenile Delinquency, and Victimization: Results from the International Self-Report Delinquency Study - 3 in 23 Countries. European Journal on Criminal Policy and Research.

De Buck, A., \& Pauwels, L. J. R. (2018). Intention to shoplift: On the importance of dimensions of propensity in an integrated informal control/lifestyle model. European Journal on Criminal Policy and Research.

Enzmann, D., Kivivuori, J., Marshall, I. H., Steketee, M., Hough, M., \& Killias, M. (2018). A Global Perspective on Young People as Offenders and Victims (First Results from the ISRD3 Study). Cham, Switzerland.

Enzmann, D., Marshall, I. H., Killias, M., Junger-Tas, J., Steketee, M., \& Gruszczynska, B. (2015). Second International Self-Reported Delinquency Study, 2005-2007. Ann Arbor: Inter-university Consortium for Political and Social Research [distributor].

Gottfredson, M. (2018). Foreword. In D. Enzmann, J. Kivivuori, I. H. Marshall, M. Steketee, M. Hough, \& M. Killias (Eds.), A Global Perspective on Young Offenders and Victims (First Results from the Isrd3 Study) (pp. v-v111). New York: Springer.

Junger-Tas, J., Marshall, I. H., Enzmann, D., Killias, M., Steketee, M., \& Gruszczynska, B. (2012). The Many Faces of Youth Crime. Contrasting Theoretical Perspectives on Juvenile Delinquency across Countries and Cultures. New York: Springer.

Junger-Tas, J., Marshall, I. H., \& Ribeaud, D. (2003). Delinquency in an international Perspective - The international Self-Report Delinquency Study. Amsterdam: Criminal Justice Press/ Kugler.

Kammigan, I., Enzmann, D., \& Pauwels, L. J. R. (2018). Over- and underreporting of drug use: A cross-national inquiry of social desirability through the lens of situational action theory. European Journal on Criminal Policy and Research.

Manzoni, P., \& Schwarzenegger, C. (2018). The Influence of Earlier Parental Violence on Juvenile Delinquency: The Role of Social Bonds, Self-Control, Delinquent Peer Association and Moral Values as Mediators. European Journal on Criminal Policy and Research.

Marshall, I. H. (2010). "Pourquoi Pas?" Versus "Absolutely Not!”. Cross-National Differences in Access to Schools and Pupils for Survey Research. European Journal on Criminal Policy and Research, 16(2), 89109.

Marshall, I. H., \& Boutellier, H. (2013a). Special Issue on Compassionate Criminology: The Legacy of Josine Junger-Tas. European Journal of Criminal Policy and Research, 19(2).

Marshall, I. H., \& Boutellier, H. (2013b). Josine Junger-Tas: The Life and Works of a Compassionate Criminologist. European Journal on Criminal Policy and Research, 19(2), 71-84.

Marshall, I. H., \& Enzmann, D. (2012). Methodology and Design of the ISRD-2 Study. In J. Junger-Tas, I. H. Marshall, D. Enzmann, M. Killias, M. Steketee, \& B. Gruszczynska (Eds.), The Many Faces of Youth Crime. Contrasting Theoretical Perspectives on Juvenile Delinquency across Countries and Cultures (pp. 21-65). New York: Springer.

Martins, P. C., Mendes, S. M., Fernández-Pacheco, G., \& Tendais, I. (2018). Juvenile Victimization in Portugal through the Lens of ISRD-3: Lifetime Prevalence, Predictors, and Implications. European Journal on Criminal Policy and Research.

Rocca, G., Verde, A., \& Gatti, U. (2019). Impact of Alcohol and Cannabis Use on Juvenile Delinquency: Results from an International Multi-City Study (ISRD3). European Journal on Criminal Policy and Research.

Rodríguez, J., Pérez-Santiago, N., \& Birkbeck, C. (2015). Surveys as cultural artefacts: Applying the International Self-Report Delinquency Study to Latin American adolescents. European Journal of Criminology, 12(4), 420-436. https://doi.org/10.1177/1477370815581701.

Yu, J. W., Tian, G. L., \& Tang, M. L. (2008). Two new models for survey sampling with sensitive characteristics: Design and analysis. Metrika, 67, 251-263.

Publisher's Note Springer Nature remains neutral with regard to jurisdictional claims in published maps and institutional affiliations. 